Research Article

\title{
Global Stability and Dynamic Analysis for a Type of Macroeconomic Systems
}

\author{
Ya-Juan Yang $\mathbb{D}^{1,2}$ Ru-Fei Ma, ${ }^{1}$ and Jing Zhang $^{3}$ \\ ${ }^{1}$ School of Business, Macau University of Science and Technology, Taipa, Macau \\ ${ }^{2}$ City College, Dongguan University of Technology, Dongguan, Guangdong, China \\ ${ }^{3}$ Department of Continuing Education, Dong Guan Polytechnic College, Dong Guan, China \\ Correspondence should be addressed to Ya-Juan Yang; 1909853gbm30002@student.must.edu.mo
}

Received 26 June 2020; Accepted 29 July 2020; Published 28 August 2020

Guest Editor: Fuqiang Gu

Copyright ( 92020 Ya-Juan Yang et al. This is an open access article distributed under the Creative Commons Attribution License, which permits unrestricted use, distribution, and reproduction in any medium, provided the original work is properly cited.

This paper aims at the dynamic properties of the proposed globally planned economic systems named after CPE proposed by LooKeng Hua who is one of the worldwide famous Chinese mathematicians. First, we give new existence conditions of growth balanced solution to the model. Second, we lead into the concept of stability for balanced output and carry out a theorem that deals with some equivalent conditions for judging a solution of output starting from the fact that any initial input can whether approach the existing balanced solution or not. Third, a new dynamic price system related to interest factors is proposed here and it is demonstrated that the new price equation is a much generalized one in comparison with the original price one which is only a special case of this new price equation. Also, relationships of the balanced solutions between the price and the output equation are investigated and the stability analysis is studied as well for the new price system. Finally, two examples are employed to illustrate the technical operation of input-output method and some new contributions of this article.

\section{Introduction}

In the last century, undoubtedly, the most widely used economic theory is the input-output method which was proposed by Wassily Leontief, a winner of the Nobel Prize in economics in the year 1973. The seminal paper for the inputoutput method originated in 1953 by Leontief (see [1]) and the dynamic analysis for these types of economic systems was investigated in his two classical papers $[2,3]$. A systematic discussion for input-output theory can be found in Leontief s book [4] and more references therein. Just as this method's validity in analyzing a country's economic development, there had been over 80 counties which prepared and reported their input-output economy statements by the input-output theory since 1979. Meanwhile, the United Nation's social and economic sections suggest its member that the input-output method should be led into the national statistics accounting systems.

Research of the input-output method for fully free competition market economy, FFCME in short, has received tremendous attention in the past six decades from the first proposal by Leontief and massive literature has been published since then. All these research articles, of the most important ones presented here, focused on various sorts of FFCME problems that could be typically classified into the following two types: one investigated the dynamical properties for Leontief's model (see [5-9]) and the other focused on the macroforecasting problems of the FFCME systems (see [10-13]). Status, progress, and application fields of Leontief's theory can be found in the latest symposium by Peterson (see [14]) while a complete and standard treatment to the theory here recommends to read the book by Miller and Blair [15].

Different from the FFCME system, there is another economic system named after centrally planned economy, $\mathrm{CPE}$ in short, characterized by central planning being chief while the market readjustment being accessorial and it has been executed in China over 60 years since the creation of PRC: exceeding 30 years, going on from 1949 to 1980, for complete CPE and 37 years for socialist market economy, 
beginning from 1981. For the CPE system, Leontief accompanied President Nixon in an advisory capacity at the Beijing interview of President Nixon with Chairman Mao in 1987 and he then gave a high appraisal to the policy of the socialist market economy in his review article "socialism is practicable in China" when he came back to America. The input-output technology owns a wide application in the macroforecasting and planning of economic problems regardless of short term or long term as well as the ability to be applied to forecast and plan different economic policy systems, being whether the FFCME ones or CPE ones.

Compared with the massive research literature to the FFCME system, there has been much less research focusing on the CPE system until Professor Loo-Keng Hua, a world famous mathematician of China, starting with a seminal paper in USA [16], published a series of articles in Chinese Science Bulletin at the middle of 80 s last century; see [17-23]. Of course, Professor Loo-Keng Hua's research extends Leontief's theory to the CPE system which is put into practice by China and hence is more suitable for analyzing the current economic development in China. Fundamentals of Professor Loo-Keng Hua's analysis are based on a hypothesis that China's productivity elasticity is large enough; it is allowable as Professor Blanchard points out in [24], so that the relationships between inputs and outputs can be then described well for the CPE system.

Although Professor Loo-Keng Hua established the mathematical models for analyzing the CPE system in articles as mentioned above and also some basic concepts and analysis, such as superior limit of production capacity, crisis of production system, price, and consuming coefficients, were introduced there; there are still many problems, especially dynamical properties, which need to be further investigated, for instance, the existence of this CPE system's balanced solution and its stability and price dynamics.

Motivated by this idea and in order to be in a parallel way in studying dynamical properties of the FFCME system $[2,3,5-9]$, this paper first presents the existence of growth balanced solutions and their stabilities to the CPE system, and, then, a generalized dynamic price system is proposed with key merit that Loo-Keng Hua's primary price system is a special case of this. Meanwhile, relationships of the balanced solution between price and output are analyzed as well as the stability of the dynamic price system investigated too.

The remainder of this paper is organized as follows: Section 2 is an outline of CPE system formulation and some results of the study being made by Professor Loo-Keng Hua are reviewed there. The main study of this economic system in this paper is carried out in the next sections: new existence theorem for the growth balanced solution is addressed in Section 3 and the stability analysis for the output balance is in Section 4. Section 5 deals with the dynamic price system including the formulation and the stability of balanced price while Section 6 gives two examples for supporting our theoretic results. Finally, some concluding remarks are drawn in Section 7.

Remark 1. The so-called FFCME, also known as free enterprise economy, in which the production and sale of goods and services are completely guided by the free price mechanism of fully free competition market. Up to now, absolute fully free competition market economies do not exist, but their markets are open wider than others. The United States is generally regarded as the representative of the fully free competition market economy for it's higher market access. Typical examples of CPMs are the Soviet Union and China from 1949 to 1978. Actually, in today's world, many countries conduct "more than half" of fully free competition economies, such as today's China.

\section{CPE Model and Leontief Model}

In Leontief's macroeconomic model, the production activities of an economy are divided into $n$ industrial sectors and product transactions between these sectors are analyzed based on the following basic assumptions:

$\mathrm{H} 1$ : there is no joint production, and each industry sector produces only one product, which means there is a one-to-one correspondence between sectors and products, and so they can be substituted for each other.

$\mathrm{H} 2$ : a single product produced by each sector requires a certain amount of input from other sectors; that is, sector $j$ requires $t_{i j}$ units of product $i$ as input to produce a unit of the $j$ th product; here $i=1,2, \ldots, n$ and $t_{i j}$ are called the input coefficients.

H3: in the whole production process of an economy, there is no lag in production, no capital goods, no foreign trade, neither involvement of government activities.

Under the above assumptions, let $x_{i}$ be the total output of the $j$ th product and $x=\left(x_{1}, x_{2}, \ldots, x_{n}\right)^{T}$ the output vector. For production of the other $n$ sectors, the amount consumed by $x_{i}$ is

$$
\sum_{j=1}^{n} t_{i j} x_{i},
$$

and the remaining amount will be

$$
d_{i}=x_{i}-\sum_{j=1}^{n} t_{i j} x_{i}
$$

This net output $d_{i}$ is called the final demand of the $i$ th product, and let $d=\left(d_{1}, d_{2}, \ldots, d_{n}\right)^{T}$ represent the final demand vector.

Then, as the aggregate demand must be equal to the aggregate supply, we can get the following total input-output equation systems:

$$
(I-T) x=d,
$$

which describe the economy as a whole; here, $T=\left(t_{i j}\right) \in M_{n}(R)$ is called the input matrix and $L=I-T$ the Leontief matrix. These input-output systems (3) are called open Leontief model and they are the theoretical basis for analyzing the macroeconomic state of an economy.

Leontief s method gives an important contribution in the optimization of the entire production process since it is a very complex problem and there has not been a corresponding mathematical method which can fully solve this optimal task, especially the lack of mathematical description 
for the large-scale economic behavior. The hypotheses (H1)(H3) of Leontief's model are very strict ones (only can be partially meted by FFCME as mentioned in Section 1), which are far from the actual economic operation state, but this does not affect the wide application of the input-output analysis for an economy.

Why do we say that? The most important economic activity in an economy is the production and the domestic sales and hence what we are interested in here are the economy's intersectoral input and the external demand, this may be the reason that foreign trade is excluded in hypothesis 3. Because Leontief's method just meets this purpose, the input-output reports have been employed by, as mentioned earlier, more than 80 countries in the world. While carrying out an economy's input-output report, there are many various input-output tables to be created and details for making an input-output table can be found in Section 6; see Example 1 therein.

Although the input-output analysis has become the standard template for much national economic planning, national development plans cover much more than economics such as population, welfare, and environment. For a country, an economy, taking these problems together, boils down to a multiobjective decision problem of which the first study is made by Schinnar who analyzes development planning in a Leontief input-output model in order to take into account both economic and demographic goals; see [25].

Thereafter, Chichilnisky carried out first the green golden rule in respect of social welfare and then defines social welfare as a weighted average between the discounted utilitarianism and the green golden rule welfare; see [26, 27]. Up to now, we believe that Leontief's model combined with the multigoal decision-making model will be the most promising research topics for addressing macroeconomic planning and forecasting, for which the interested readers may refer to the review article [28] by Cinzia et al.

Different from Leontief's input-output model, Professor Loo-Keng Hua proposed a CPE system model (see $[17,18]$ ), where the national production is divided into $n$ sectors also, which are interrelated with a so-called consumption coefficient matrix $A=\left(a_{i j}\right)_{n \times n}$, with $a_{i j}$ being the quantities of the $j$ th class to be consumed for producing a unit quantity of the $i$ th class. It is obvious that $A=\left(a_{i j}\right)_{n \times n} \geq 0$ and $a_{i j}>0$ for some $i, j$.

By letting $X^{t}$, normally being a $n$-dimensional column vector, be the output of the $t$-period, Loo-Keng Hua established the CPE system as follows:

$$
(\mathrm{CPE}) X^{t}=A X^{t+1}, \quad t \in T=\{0,1,2, \ldots\} .
$$

If $A$ is an invertible matrix, the previous equation, equivalently, can be formulated by

$$
\text { (CPE) } X^{t+1}=A^{-1} X^{t}, \quad t \in T=\{0,1,2, \ldots\} .
$$

This CPE system is based on the assumption that the production of every class grows in a fixed proportion at each period $t$ and the consumption coefficient matrix does not vary with time $t$. It is easy to see, by the inductive method, that $X^{(t)}=\left(1 / \lambda^{t}\right) X^{(0)}$ will be the $t$ - period production, namely, a solution of the CPE system (4) or (5) if the initial input starting from an eigenvector $X^{(0)}>0$ of $A$ with the corresponding eigenvalue $\lambda$.

It should be noted that if we let $t=0$, by (4), then $X^{0}=$ $\mathrm{AX}^{1}$; substituting it to $X^{1}-X^{0}$ gives us

$$
X^{1}-X^{0}=(I-A) X^{1},
$$

which is the same as Leontief s model (3) with $d=X^{1}-X^{0}$, $x=X^{1}$, and $T=A$. So, in this sense, Leontief's model is a special case of the proposed CPE model.

At the same time, Loo-Keng Hua also gave a price equation coupled with equation (4) as follows:

$$
\left(q_{1}, q_{2}, \ldots, q_{n}\right) \lambda_{*}=\left(q_{1}, q_{2}, \ldots, q_{n}\right) A,
$$

where $q_{i}$ is the price per-unit of the $i$ th class and $\lambda_{*}$, also called the "price changing rate," is the largest eigenvalue of $A$. According to Loo-Keng Hua's primary definition, the price defined here means the product's basic output value only without the profits being contained; see [20].

For the CPE system (4), Loo-Keng Hua investigated the fundamental dynamical property (see Theorem 1 of [18]), where it is called "basic theorem" and we keep it here.

Basic Theorem: let $A$ be a nonnegative irreducible square matrix, for any noneigenvector of $A$ but positive vector $x>0$; there exists a positive integer $l_{0}$ such that

$$
x A^{-l}
$$

must be variable vector when $l \geq l_{0}$, namely, some entries of $x A^{-l}$ are positive and some negative.

It follows from this Basic Theorem that some outputs of production will be negative in several years if the initial input is a positive noneigenvector of $A$ and, thus, the economic system will collapse. So, the Basic Theorem reveals a basic principle of philosophy that balance is temporary but imbalance is perpetual for any economic system: imbalance will ultimately happen and even collapse, someday if the initial input $x$ is not an eigenvector of the consumption coefficient matrix $A$.

Of course, it is far from enough to stop at this Basic Theorem for the dynamical properties of the CPE system (4) as a much deeper study with respect to the dynamical property for the FFCME system is investigated by $[2,3,5,25]$.

To proceed, we need some concepts of dynamical property for economic systems such as definitions of the balanced solution and the causal indeterminacy proposed by Solow and Samuelson in [29].

Definition 1. For the CPE system (4), if there exists a constant $\alpha>0$ such that $X^{t}=\alpha X^{t-1}$ for all $t \in N=\{1,2, \cdots\}$, then $X^{t}$ is called a balanced solution of (4) with the growth rate $\alpha$.

It is clear that if the consumption coefficient matrix $A$ is a nonnegative irreducible square matrix, then, by the wellknown Perron-Frobenius theorem, there exists a positive eigenvector $X$ of $A$ that must be a balanced solution of the CPE system (4) with the growth rate $\left(1 / \lambda_{*}\right)$; here $\lambda_{*}$ is the largest positive eigenvalue of $A$ and $X$, being the 
corresponding eigenvector. Obviously, by Definition 1, the economic quantity of the CPE system (4) will increase as $\left(1 / \lambda_{*}>1\right)$ and decrease as $\left(0<1 / \lambda_{*}<1\right)$.

The case occurring in the Basic Theorem is called the causal indeterminacy for an economic system as defined in the following definition.

Definition 2. If the initial input of an economic system does not meet any possible balanced solution, then there must exist at least one output to be negative. The economic system that owns this property will be known as having the causal indeterminacy.

Remark 2. By the Basic Theorem, it follows that the causal indeterminacy must happen for the CPE system (4) if the consumption coefficient matrix $A$ is a nonnegative irreducible square matrix.

\section{Existence of the Growth Solution}

As stated in Remark 2 of the previous section, the CPE system (4) yields a balanced solution with the economic growth rate $\left(1 / \lambda_{*}\right)$, if the consumption coefficient matrix $A$ is a nonnegative irreducible square matrix, thus the economic system will develop well when $\left(1 / \lambda_{*}>1\right)$ since the economic growth keeps on. It is certain that there are some other existence conditions on the balanced growth solution of the CPE system (4) except for the ones proposed by LooKeng Hua and it would be best to find these conditions for the existence of the growth balanced solution so as to control the economic system developing under the way. Meanwhile, this section will focus on these themes then.

Different from Loo-Keng Hua's assumption on the consumption coefficient matrix $A$ being an irreducible matrix, here we try to find the existence condition for the more general matrix ones. First, we need two lemmas for proving the main existence theorem.

Lemma 1. Let $A=\left(a_{i j}\right)_{n \times n}$ be a nonnegative real matrix with a nonnegative real eigenvalue $\lambda \geq 0$ that dominates all the other eigenvalues $\lambda_{i}(i=1,2, \ldots)$ of $A$ in absolute value, that is, $\lambda \geq\left|\lambda_{i}\right|, i=1,2, \ldots$; then, for any positive vector $x=\left(x_{1}, x_{2}, \ldots, x_{n}\right)^{T}$, we have

$$
\min _{1 \leq i \leq n}\left(\frac{1}{x_{j}} \sum_{j=1}^{n} a_{i j} x_{j}\right) \leq \lambda \leq \max _{1 \leq i \leq n}\left(\frac{1}{x_{j}} \sum_{j=1}^{n} a_{i j} x_{j}\right) .
$$

Proof. See [30] Theorem 8.1.26.

Lemma 2. Suppose $A$ is the consumption coefficient matrix of CPE system (4) and $I-A$ is a diagonal strictly dominant matrix; then there exist a positive real number $\mu$ : $0<\tilde{\mu}<1$ and a positive vector $\tilde{x} \geq 0$ such that $A \tilde{x}=\tilde{\mu} \tilde{x}$.

Proof. Since $A$ is the consumption coefficient matrix of CPE system (4), we have $A \geq 0$. Then, it follows by the well-known Perron-Frobenius theorem that there exist a positive real number $0 \leq \tilde{\mu} \leq 1$ and a positive vector $\tilde{x} \geq 0$ such that $A \tilde{x}=\tilde{\mu} \tilde{x}$.

By Gershgorin theorem, all the eigenvalues of $A$ belong to the set

$$
\bigcup_{i} \Omega_{i}=\left\{\mu\left|\mu-a_{i i}\right| \leq \sum_{j \neq i} a_{i j}\right\} .
$$

Thus, we have $\left|\widetilde{\mu}-t a_{i i}\right| \leq \sum_{j \neq i} a_{i j}$.

By this and the assumption of $I-A$ being a diagonal strictly dominant matrix, that is, $1-a_{i i}>\sum_{j \neq i} a_{i j}$, we get $2 a_{i i}-1<\widetilde{\mu}<1$ and hence $\widetilde{\mu}<1$.

In addition, according to Lemma 1, by letting $x=\left(x_{1}, x_{2}, \ldots, x_{n}\right)^{T}=(1,1, \ldots, 1)^{T}$, we get

$$
\min _{1 \leq i \leq n}\left(\sum_{j=1}^{n} a_{i j}\right) \leq \tilde{\mu} \leq \max _{1 \leq i \leq n}\left(\sum_{j=1}^{n} a_{i j}\right) .
$$

So, according to the nonnegative of $A$, namely, $\sum_{j=1}^{n} a_{i j} \geq 0$, as well as the economic meaning of $A$, we get that $\sum_{j=1}^{h} a_{i j} \geq 0$ and hence $\tilde{\mu}>0$. Therefore, it follows that there exist $\tilde{\mu}$ : $0<\tilde{\mu}<1$ and a vector $\tilde{x} \geq 0$ such that $A \widetilde{x}=\tilde{\mu} \tilde{x}$.

Now, we can give the main result about the existence of growth balanced solution for the CPE system (4).

Theorem 1. Let $A=\left(a_{i j}\right)_{n \times n}$ be the consumption coefficient matrix of CPE system (4) with the assumption of $1-a_{i i}>\sum_{j \neq i} a_{i j}$; it could be satisfied when $I-A$ is a diagonal strictly dominant matrix. Then, there exists a growth balanced solution $X^{(t)}=(1 / \tilde{\mu})^{t} \tilde{x}$ of system (4) with the growth rate $(1 / \widetilde{\mu})$; here $\tilde{\mu}: 0<\tilde{\mu}<1$ is the eigenvalue of $A$ and $\tilde{x} \geq 0$ the corresponding eigenvector.

Proof. First, letting $t=0$, by system (4), we get

$$
X^{0}=A X^{1} \text {. }
$$

If $X^{0}$ is a balanced solution of (12) with the growth rate $(1 / \lambda)$, then by Definition 1 , we get

$$
X^{1}=\frac{1}{\lambda} X^{0}
$$

It follows from (12) and (13) that

$$
\lambda X^{0}=\mathrm{AX}^{0}
$$

Inductively, it is easy to get that $X^{t}=(1 / \lambda)^{t} X^{0}$ will also be a balanced solution.

Second, by letting $X^{0}=\tilde{x}$ and according to Lemma 2, we obtain that $X^{(t)}=(1 / \tilde{\mu})^{t} \tilde{x}$ will be a growth balanced solution with growth rate $(1 / \tilde{\mu})>1$. Therefore, Theorem 1 is proved then.

Remark 3. Theorem 1 gets rid of the restriction that the consumption coefficient matrix should be irreducible. Further, under the condition proposed here, it is impossible that the total costs in quantities for each class will be more than the total outputs to be produced. This is the reason for the occurrence of negative output then. So, controlling the 
economic system (4) in this way will be a better choice since there is no causal indeterminacy occurring under this direction for the CPE system (4).

\section{Stability of the Balanced Output}

As stated in the previous section, a better way to control an economic system is to avoid the causal indeterminacy occurring and find the growth solution so as to keep the economy growing. Though Theorem 1 gives a good description of finding out the growth balanced solution, the condition for this needs a rigid initial point so as to reach the goal.

If started at any point, it is vitally important to investigate whether this solution can approach the existing growth balanced solution or not, namely, the stability of the economy balance. First, we need a mathematical description for the stability; see the following definition.

Definition 3. Let $A \geq 0$ be the consumption coefficient matrix of system (4) and assume it is an invertible matrix. Suppose $X^{*(t)}=\left(1 / \mu^{t}\right) X>0$ is a balanced solution of system (4); if for every solution $\widehat{X}^{(t)}$ starting from any initial input $\widehat{X}^{(0)} \geq 0$, there exists a constant $\sigma: 0<\sigma<\infty$ such that $\lim _{t \rightarrow \infty}\left(\hat{x}_{i}^{(t)} / x_{i}^{*(t)}\right)=\sigma$, where $\hat{x}_{i}^{(t)}, x_{i}^{*(t)}$ is the $i$ th entry of $\hat{X}^{(t)}$ and $X^{*(t)}$, respectively, then the balanced solution $X^{*(t)}$ is called a stable balanced solution.

Clearly, if the CPE system (4) yields a stable balanced solution, then all the solutions determined by any initial input will eventually be greater than zero since any of these solutions approaches asymptotically to the positive balanced solution $X^{*(t)}>0$. Thus, there has been no causal indeterminacy happening in this case for system (4).

Next, we give a necessary and sufficient condition to the stability of a balanced solution for system (4).

Theorem 2. Suppose $A \geq 0$ is the consumption coefficient matrix of system (4) as well as an invertible matrix; then we have the following:

(i) There exists a positive integer $t$ such that $\left(A^{-1}\right)^{t}>0$ if and only if $\left|\lambda_{i}\right|>\lambda_{1}>0, X_{1}>0$, where $\lambda_{i}, i=$ $1,2, \ldots, n$ are the eigenvalues of $A$ and $X_{i}$ the corresponding eigenvectors.

(ii) The balanced solution $X^{*(t)}=\left(1 / \lambda_{1}^{t}\right) X_{1}>0$ is stable if and only if $\left|\lambda_{i}\right|>\lambda_{1}>0$, where $\lambda_{i}, i=1,2, \ldots, n$ are the eigenvalues of $A$ and $X_{i}$ the corresponding eigenvectors.

Proof. First of all, let us prove that (i) for any invertible matrix $A \geq 0$, there exists a positive integer $t$ such that $\left(A^{-1}\right)^{t}>0$ if and only if $\left|\lambda_{i}\right|>\lambda_{1}>0, X_{1}>0$, where $\lambda_{i}, i=1,2, \ldots, n$ are the eigenvalues of $A$ and $X_{i}$ the corresponding eigenvectors.

If $\left(A^{-1}\right)^{t}>0$ for some positive $t$, then $\left(A^{-1}\right)^{t}>0$ will be an irreducible matrix. It follows by the well-known Frobenius theorem that matrix $\left(\left(A^{-1}\right)^{t}\right)^{\prime} s$ eigenvalues $\mu_{i}$ and the corresponding eigenvectors $Y_{i}(i=1,2, \ldots, n)$ must satisfy $\mu_{1}>\left|\mu_{i}\right|, Y_{1}>0$ with $\mathrm{AY}_{1}=\mu_{1} Y_{1}$, where, $i=2,3, \ldots, n$. Let $\lambda_{i}$ be the eigenvalues of $A$ and $X_{i}$ the corresponding eigenvectors; then it is easy to say $\mu_{i}=\left(1 / \lambda_{i}^{t}\right), i=1,2, \ldots, n, Y_{1}=X_{1}$. So, by $\mu_{1}>\left|\mu_{i}\right|$, we get $\left(1 / \lambda_{1}^{t}\right)>\left|\left(1 / \lambda_{i}^{t}\right)\right|(i=2,3, \ldots, n)$ and hence $\left|\lambda_{i}\right|>\lambda_{1}>0, X_{1}>0$.

On the contrary, let $\lambda_{i}$ be the eigenvalues of $A$ and $X_{i}$ the corresponding eigenvectors with $\left|\lambda_{i}\right|>\lambda_{1}>0, X_{1}>0$, where $i=2,3, \ldots, n$. First, any vector $V$ could be represented as a linear combination of $X_{i}$ for the invertibility of $X_{i}$; that is,

$$
V=h_{1} X_{1}+h_{2} X_{2}+\cdots+h_{n} X_{n}
$$

Let $V=E^{i}$ be the column vector of the unit matrix $E$, that is, all the entries of $E^{i}$ being 0 except for the ith being 1; then we have

$$
E^{i}=h_{1} X_{1}+h_{2} X_{2}+\cdots+h_{n} X_{n}
$$

Making the inner product of this $E^{i}$ with $X_{1}$, we get

$$
\left[X_{1}, E^{i}\right]=\left[X_{1}, h_{1} X_{1}+h_{2} X_{2}+\cdots+h_{n} X_{n}\right]=\sum_{i=1}^{n} h_{i}\left[X_{1}, X_{i}\right]
$$

Note that $\left[X_{1}, X_{i}\right]=0$ for $i \neq 1$; we have $\left[X_{1}, E^{i}\right]=h_{1}\left[X_{1}, X_{1}\right]$ and so $h_{1}=\left(\left[X_{1}, E^{i}\right] /\left[X_{1}, X_{1}\right]\right) . h_{1}$ must be positive since $X_{1}>0$ and the choice of $E^{i}$.

Similarly, any solution $\hat{X}^{(t)}$ with starting initial input $E^{i}$ for system (5) can be reformulated as

$$
\widehat{X}^{(t)}=\left(A^{-1}\right)^{t} E^{i}=h_{1} \frac{1}{\lambda_{1}^{t}} X_{1}+h_{2} \frac{1}{\lambda_{2}^{t}} X_{2}+\cdots+h_{n} \frac{1}{\lambda_{n}^{t}} X_{n},
$$

or, equivalently,

$$
\lambda_{1}^{t} \widehat{X}^{(t)}=\lambda_{1}^{t}\left(A^{-1}\right)^{t} E^{i}=h_{1} X_{1}+h_{2} \frac{\lambda_{1}^{t}}{\lambda_{2}^{t}} X_{2}+\cdots+h_{n} \frac{\lambda_{1}^{t}}{\lambda_{n}^{t}} X_{n} .
$$

By $\left|\lambda_{i}\right|>\lambda_{1}>0$, that is, $0<\left(\lambda_{1} /\left|\lambda_{i}\right|\right)<1$, and paying attention to $X_{1}>0, h_{1}>0$, we get that, for sufficient enough, large $t \geq N$,

$$
\lambda_{1}^{t} \widehat{X}^{(t)}=\lambda_{1}^{t}\left(A^{-1}\right)^{t} E^{i}>0 .
$$

As $\lambda_{1}^{t}>0$, we obtain $\left(A^{-1}\right)^{t} E^{i}>0$ for large $t$. Since it holds for any $E^{i}$, we get that $\left(A^{-1}\right)^{t}>0$.

Secondly, we prove (ii). If $\left|\lambda_{i}\right|>\lambda_{1}>0$, according to Definition 3 and (18) we have

$$
\begin{aligned}
\lim _{t \rightarrow \infty} \frac{\hat{x}_{i}^{(t)}}{x_{i}^{*(t)}} & =\frac{h_{1}\left(1 / \lambda_{1}^{t}\right) X_{1 i}+h_{2}\left(1 / \lambda_{2}^{t}\right) X_{2 i}+\cdots+h_{n}\left(1 / \lambda_{n}^{t}\right) X_{n i}}{\left(1 / \lambda_{1}^{t}\right) X_{1 i}} \\
& =h_{1} .
\end{aligned}
$$

Let $\sigma=h_{1}$ and note that $h_{1}>0$; we get that this $X^{*(t)}$ should be a stable balanced solution.

On the other hand, if $\lim _{t \longrightarrow \infty}\left(\widehat{x}_{i}^{(t)} / x_{i}^{*(t)}\right)=\sigma>0$, then, for sufficiently large $t \geq N$, we have $\hat{x}_{i}^{(t)}>0, i=1,2, \ldots, n$. So, $\widehat{X}^{(t)}>0$ for sufficiently large $t \geq N$ with any initial input vector $\widehat{X}^{0} \geq 0$. Similar to the previous proof of (i), if we let 
$\widehat{X}^{0}=E^{i}$, then we obtain $\widehat{X}^{(t)}=\left(A^{-1}\right)^{t} E^{i}>0$ for all $i=1,2, \ldots, n$ and sufficiently large $t \geq N$. Thus, $\left(A^{-1}\right)^{t}>0$. Therefore, by proving (i), we get that $\left|\lambda_{i}\right|>\lambda_{1}>0, X_{1}>0$.

Remark 4. Up to now, for the CPE system (4), under the assumption of the consumption coefficient matrix $A \geq 0$ being invertible, we have the following equivalent results: the balanced solution $X^{*(t)}=\left(1 / \lambda_{1}^{t}\right) X_{1}>0$ is stable $\Leftrightarrow$ there exists a positive integer $t$ such that $\left(A^{-1}\right)^{t}>0 \Leftrightarrow\left|\lambda_{i}\right|>$ $\lambda_{1}>0, X_{1}>0 \Leftrightarrow$ causal indeterminacy cannot occur $\Leftrightarrow$ each product's output cannot be negative in the subsequent years later.

\section{Dynamic Price System}

Loo-Keng Hua's price equation (7) is based on not considering the capital costs such as the money rate. At the same time, it gives an assumption that the product's price changes in a fixed proportion, for instance, $\lambda^{*}$; that is $P^{(t+1)}=\lambda_{*} P^{(t)}$ for each period $t$. But, certainly, interest rate impacts directly on the product's sale price while the product's sale price may generally not change in a fixed proportion in practical economic environment. Hence, we need to rebuild or extend Loo-Keng Hua's price system so as to match the practical economic system soundly and this will be carried out in the following subsection 5.1.

In addition, subsection 5.2 deals with the dynamical properties for the proposed price system such as the balanced price solution and its stability as well. The deep research on this respect will contribute to controlling the economic system (4) in a way of keeping the economy growing continually.

5.1. Formulation of the Price System. Let $P_{i}^{(t)}$ be the price of the $i$ th class per-unit product in the $t$-period. The price vector for all $n$ class products in the $t$-period could be represented as $P^{(t)}=\left(P_{1}^{(t)}, P_{2}^{(t)}, \ldots, P_{n}^{(t)}\right)$. Assume any product's price to be a fixed constant during each period and costs of other products consumed for producing any product to be paid at the beginning of each period. Then, the per-unit cost for producing the $j$ th class product in the $t$-period is

$$
v_{j}^{(t)}=\sum_{i=1}^{n} P_{i}^{(t)} a_{i j}=P^{(t)} a_{j},
$$

where $a_{j}$ is the $j$ th column of the consumption coefficient matrix $A$.

The per-unit profit, regardless of other costs, for producing the $j$ th class product in the $t$-period will be

$$
\pi_{j}^{(t)}=P_{j}^{(t+1)}-P^{(t)} a_{j}
$$

At the same time, if money to be loaned out can get an interest rate $r^{t}$ during the time between $t$-period and $(t+1)$-period, then the capital revenue for buying $v_{j}^{(t)}$ will be

$$
R_{j}^{(t)}=r^{t} v_{j}^{(t)}=r^{t} P^{(t)} a_{j}
$$

By the competition arbitrage principle, interest and profit should eventually reach an equilibrium state; that is,

$$
\pi_{j}^{(t)}=R_{j}^{(t)}
$$

So,

$$
P_{j}^{(t+1)}-P^{(t)} a_{j}=r^{t} P^{(t)} a_{j}
$$

or

$$
P_{j}^{(t+1)}=\left(1+r^{t}\right) P^{(t)} a_{j}
$$

As (27) holds for all $j=1,2, \ldots, n$, we can formulate it in the following matrix representation:

$$
P^{(t+1)}=\left(1+r^{t}\right) P^{(t)} A
$$

Letting $M=\left(1+r^{t}\right) A$, we obtain the dynamic price equation corresponding to (4) as follows:

$$
P^{(t+1)}=P^{(t)} M
$$

If interest rate $r^{t}=0$ and the price changing rate varies with a fixed proportion $\lambda_{*}=\left(P_{i}^{t+1} / P_{i}^{t}\right)$ for each period $t$, then this dynamic price equation (29) becomes the price equation (7) proposed by Loo-Keng Hua. Next, we turn to investigate the balanced price solution and the stability for the price equation (29) as made previously for the output equation (4).

5.2. Stability of the Balanced Price. In order to simplify, we suppose the interest rate $r^{t}=r$ to be a constant and $A \geq 0$ to be invertible. It is easy to see that $M=(1+r) A$ is also invertible and the eigenvalues of $M$ will be $(1+r) \lambda_{i}$ if $\lambda_{i}$ is the eigenvalues of $A$ while the corresponding eigenvectors are the same.

Definition of the balanced price solution to (29) can be defined as Definition 1 for output equation (4); that is, if $P^{(t)}=\beta P^{(t-1)}$ holds for all $t \in N=\{1,2, \ldots, n, \ldots\}$ and some constant $\beta>0$, then $P^{(t)}$ will be a balanced solution of (29) with price changing rate $\beta$. The definition of stability for a balanced price solution can be found in the following mathematical description.

Definition 4. Let $A \geq 0$ be an invertible matrix and $M=(1+r) A$. Suppose $P^{*(t)}=\zeta_{1}^{t} p_{1}>0$ is a balanced price solution of system (29); if, for a solution $\widehat{P}^{(t)}$ starting from any initial price $\widehat{P}^{(0)} \geq 0$, there exists a constant $\sigma: 0<\sigma<\infty$ such that $\lim _{t \rightarrow \infty}\left(\widehat{p}_{i}^{(t)} / p_{i}^{*}{ }^{*(t)}\right)=\sigma$, where $Z$ is the $i$ th entry of $\widehat{P}^{(t)}$ and $P^{*(t)}$, respectively, then the balanced solution $P^{*}(t)$ is called a stable price solution. 
Similarly, we have Theorem 3 which reveals the dynamical properties for price equation (29) like Theorem 2 for the output equation (4).

Theorem 3. Suppose $A \geq 0$ to be an invertible matrix and let $M=(1+r) A$, where $r>0$ is the interest rate. Then, we have the following:

(i) There exists a positive integert such that $M^{t}>0$ if and only if $\mu_{1}>\left|\mu_{i}\right| \geq 0, p_{1}>0$, where $\mu_{i}$ and $p_{i}$, $i=1,2, \ldots, n$, are the eigenvalues of $M$ and the corresponding eigenvectors.

(ii) The balanced price $P^{*(t)}=\zeta_{1}^{t} p_{1}>0$ is stable if and only if $\zeta_{1}>\left|\zeta_{i}\right| \geq 0, p_{1}>0$, where $\zeta_{i}$ and $p_{i}$, $i=1,2, \ldots, n$, are the eigenvalues of $M$ and the corresponding eigenvectors.

Proof. The proof of this theorem is similar to Theorem 2 and a survey is given here.

First, it is noted that $A \geq 0$ and being invertible implies $M=(1+r) A \geq 0$ and being invertible also. Second, if $\mu$ is an eigenvalue of matrix $M$ with eigenvector $p$, then $\mu^{t}$ will be the eigenvalue of $M^{t}$ with the same eigenvector $p$ in regardless of scalar times. Third, inductively, it is easy to obtain $P^{(t)}=P^{(0)} M^{t}$. Finally, note that the general solution of equation (29) can be written as

$$
\widehat{P}^{(t)}=\alpha_{1} \zeta_{1}^{t} p_{1}+\alpha_{2} \zeta_{2}^{t} p_{2}+\cdots+\alpha_{n} \zeta_{n}^{t} p_{n},
$$

where $\alpha_{i}$ are constants to be determined by the initial price $\widehat{P}^{(0)}$. All these four points give the key respects to prove Theorem 3 and the procedure of the proof is omitted here.

Remark 5. The solutions $X^{*(t)}=\left(1 / \lambda_{1}^{t}\right) X_{1}$ and $P^{*(t)}=\zeta_{1}^{t} p_{1}$ are a pair of output balance and price balance by the correspondence $\zeta_{1}=(1+r) \lambda_{1}, X_{1}=p_{1}$. In fact, eigenvalues $\lambda_{i}$ of $A$ and eigenvalues $\zeta_{i}$ of $M=(1+r) A$ have a correspondence $\zeta_{i}=(1+r) \lambda_{i}$. So, if $\zeta_{1}>\left|\zeta_{i}\right|$, then $\lambda_{1}>\left|\lambda_{i}\right|$ and if $\left|\lambda_{i}\right|>\lambda_{1}$, then $\left|\zeta_{i}\right|>\zeta_{1}$. Thus, by Theorem 2 and Theorem 3, balanced price solution $P^{*(t)}$ being stable means balanced output $X^{*(t)}$ is not stable and vice versa.

\section{Illustrative Examples}

In this section, we illustrate some of the main results of this paper with two examples: one is about the input-output table formulation and the other is an application of Theorem 1 as well as a comparison declaration. First, let us give a simplified example to show the process of how to create an input-output table.

Example 1. Assuming a hypothetical economy is composed of (1) agriculture, (2) the industrial sector (manufacturing), and (3) the service provider (service), each of these departments produces only one type of product, namely, the agricultural, industrial, or service supply, and there is interdependence between and among them. Each department buys products from the other departments and sells its own products to the opposite ones, but the final product and service supply (they do not enter the production process) is used by external departments, such as consumers. The production process and the external demand are not associated with the government and there is no foreign trade; then, according to the early hypotheses $(\mathrm{H} 1)(\mathrm{H} 3)$, we can carry out a form to summarize the product and the current situation of service supply, as shown in Table 1 , where $x_{i j}$ represents products (in US \$) sold by sector $i$ to sector $j$.

The data of each row in Table 1 represents the allocation of the total output to different departments and users, while the data of each column represents the sources of department inputs required for the total output. For example, the first row shows that the total output of $100 \$$ agricultural products is assigned to $15 \$$ products for reproducing, $20 \$$ products being sold to manufacturing, $30 \$$ products to service, and the last $35 \$$ products being used to meet external demand. Similarly, it can be seen from the second column that for the total output of $200 \$$, the manufacturing needs to invest $20 \$$ of agricultural products, $10 \$$ of its own products, and $60 \$$ of service input.

For convenience of analysis, coupled with the input-output table, the so-called technology input-output table, can be constructed to show the amount that each sector, for the purpose of producing one unit of its own product, needs for consuming the other sector's. The quantity in the technical input-output table represents the input coefficient $t_{i j}$ (in Leontief's model) or consumption coefficient (in CPE model) of the economy, which can be obtained from Table 1, for example, the data of each column in Table 1 being divided by the total agriculture output 100 gives the agriculture sector's input coefficients and so on. Thus, the technology input table or consumption coefficient table is completed and shown in Table 2 .

By Table 2, the input matrix $T$ or consumption coefficient matrix $A$ should be

$$
T=A=\left[\begin{array}{lll}
0.15 & 0.10 & 0.20 \\
0.30 & 0.05 & 0.30 \\
0.30 & 0.30 & 0.00
\end{array}\right]
$$

The input-output table can comprehensively and systematically reflect the input-output relationship among all sectors of the national economy and reveal the economic and technological relations of interdependence and mutual restriction among all sectors in the production process. On the other hand, it can tell people about the output of various sectors of the national economy and how the output of these sectors is distributed to other sectors for production or to residents and society for final consumption or export abroad. Furthermore, it can tell people how each department obtains intermediate inputs and initial inputs from other departments for its own production.

Example 1 only provides the fundamental principle for the compilation of input-output table with a simple case. In general, the actual input-output table of an economy is much more complicated than this one provided and it generally includes national table, regional table, sectoral table, and joint enterprise table according to different scopes, as well as static table and dynamic table according to the model characteristics. In addition, there are input-output tables for 
TABLE 1: Input-output table.

\begin{tabular}{|c|c|c|c|c|c|}
\hline \multirow{2}{*}{ Input } & \multicolumn{5}{|c|}{ Output } \\
\hline & Agriculture & Manufacturing & Service & External demand & Total output \\
\hline Agriculture & $15\left(x_{11}\right)$ & $20\left(x_{12}\right)$ & $30\left(x_{13}\right)$ & $35\left(d_{1}\right)$ & $100\left(x_{1}\right)$ \\
\hline Manufacturing & $30\left(x_{21}\right)$ & $10\left(x_{22}\right)$ & $45\left(x_{23}\right)$ & $115\left(d_{2}\right)$ & $200\left(x_{2}\right)$ \\
\hline Service & $20\left(x_{31}\right)$ & $60\left(x_{32}\right)$ & $0\left(x_{33}\right)$ & $70\left(d_{3}\right)$ & $150\left(x_{3}\right)$ \\
\hline
\end{tabular}

TABLE 2: Technology input-output table.

\begin{tabular}{lccr}
\hline Input & & Output & \multicolumn{1}{c}{ Service } \\
\hline Agriculture & Agriculture & Manufacturing & $0.20\left(t_{13}\right)$ \\
Manufacturing & $0.15\left(t_{11}\right)$ & $0.10\left(t_{12}\right)$ & $0.30\left(t_{23}\right)$ \\
Service & $0.30\left(t_{21}\right)$ & $0.05\left(t_{22}\right)$ & $0.00\left(t_{33}\right)$ \\
\hline
\end{tabular}

studying special issues such as environmental protection, population, and resources.

The function of a nation's input-output report is not only to reflect the direct and obvious economic and technological relations among various departments in the production process, but also to reveal the indirect, hidden, and even neglected economic and technological relations among various departments. The input-output table provides the basis for studying the industrial structure, especially for making and checking the national economic plan, studying the price decision, and conducting various quantitative analyses.

Example 2. Let $A$ be a matrix like the following:

$$
A=\left[\begin{array}{ccc}
\frac{1}{10} & 0 & \frac{2}{5} \\
0 & \frac{1}{5} & 0 \\
0 & 0 & \frac{2}{5}
\end{array}\right],
$$

and it is easy to find the inverse of $A$ as follows:

$$
B=A^{-1}=\left[\begin{array}{ccc}
10 & 0 & -10 \\
0 & 5 & 0 \\
0 & 0 & \frac{5}{2}
\end{array}\right] .
$$

A simple computation gives us the following eigenvectors $X$ and the corresponding eigenvalues $\lambda_{i}(i=1,2,3)$ of $A$ :

$$
\begin{aligned}
& X=\left[\begin{array}{lll}
1 & 0 & \frac{4}{5} \\
0 & 1 & 0 \\
0 & 0 & \frac{3}{5}
\end{array}\right], \\
& \lambda=\left(\lambda_{1}, \lambda_{2}, \lambda_{3}\right)=\left(\frac{1}{10}, \frac{1}{5}, \frac{2}{5}\right) .
\end{aligned}
$$

It is easy to check that $1-a_{i i}>\sum_{j \neq i} a_{i j}, i, j=1,2,3$; this means the conditions of Theorem 1 are satisfied and then, by Theorem 1, CPE system (4) with consumption coefficient matrix $A$ must have a growth balanced solution. One thing that needs special attention, here, is that the matrix $A$ is reducible which is different from the fact that most of the existing dynamic properties for CPE and FFCME are based on the hypothesis of matrix $A$ to be irreducible.

\section{Conclusion}

Other than the "planned economic" policy that had been put into practice in China for nearly 30 years from 1949 to 1978, due to the first industrial revolution which started at the sixties of the $18^{\text {th }}$ century, there is the "fully free competition marketing economic" policy being conducted by the West for over one hundred years. There have been a tremendous number of research papers investigating various economic behaviors of the fully free competition marketing systems in the past hundred years but few studies investigated the planned economic systems till Professor Loo-Keng Hua, a world famous Chinese mathematician, presented a series of research papers in the 80 s of the last century. 
In parallel with the extensive investigations being done for various FFCME systems and motivated by studying along the direction of dynamical properties of a system, we have carried out some important dynamical properties for CPE system which were first proposed by the famous Chinese mathematician Loo-Keng Hua in this paper.

The main results obtained here include the followings: first, we obtain new conditions for the existence of growth balanced solution to the CPE system and investigate the stability of the balanced output as well; second, we propose a more generalized price equation and study the stability of the balanced price as well. All these results provide effective aspects for controlling the CPE system so as to keep the economy grow and develop stably.

Certainly, further study of the CPE system should be encouraged in two directions: the first one is that more suitable model is needed to match the economy developing state under the varying economy policy; for instance, each class's producing rate may be different but, here, in case of simplicity, with a hypothesis being the same growing rate; the second is that a deeper study, especially, the simulation of any economic model with the practical economy situation, will be more competitive than others. Just for this reason, we will aim at these problems in our future study on the economic systems.

\section{Data Availability}

No data were used for this paper.

\section{Conflicts of Interest}

The authors declare that they have no conflicts of interest.

\section{Acknowledgments}

The research was supported by Grant GD17XYJ29 from the Office of Philosophy and Social Science Research Project of Guang Dong Province, China.

\section{References}

[1] W. Leontief, "Dynamic Analysis in Studies in the Structure ofthe American Economy: Theoretical and Empirical ExplorationsInpinput-Output Analysis," in Isard and Helen Kistin, W. Leontief, H. B. Chenery, P. G. Clark et al., Eds., pp. 53-90, Oxford University Press, Oxford, UK, 1953..

[2] W. Leontief, "Lags and the stability of dynamic systems," Econometrica, vol. 29, no. 4, pp. 659-669, 1961.

[3] W. Leontief, "Lags and the stability of dynamic systems: a rejoinder," Econometrica, vol. 29, no. 4, pp. 674-675, 1961.

[4] W. Leontief, Input-output Economics, Oxford University Press, Oxford, UK, 1966.

[5] J. D. Sargan, "The instability of the Leontief dynamic model," Econometrica, vol. 26, no. 3, pp. 381-392, 1958.

[6] W. Leontief, "The dynamic inverse," in Proceedings of the Contributions to input-output analysis: proceedings of the fourth international conference on input-output techniques, North Holland, Geneva, Amsterdam, January 1970.

[7] A. Brody and A. P. Carter, "Inputcoutput techniques," in Proceedings of the Fifth International Conference on
inputCoutput Techniques, North Holland, Geneva, Amsterdam, January 1972.

[8] A. P. Schinnar, "The Leontief dynamic generalized inverse," The Quarterly Journal of Economics, vol. 92, no. 4, pp. 641-652, 1978.

[9] J. Tsukui and Y. Murakami, Turnpike Optimality in InputOutput Systems, North Holland, Geneva, Amsterdam, 1979.

[10] R. S. Preston, "The wharton long-term model: input-output within the context of a macro forecasting model," Econometric Model Performance: Comparative Simulations of the U.S. economy, pp. 271-287, University of Pennsylvania Press, Philadelphia, PA, USA, 1976.

[11] W. Leontief, A. P. Carter, and P. A. Petri, The Future of the World Economy: A United Nations Study, Oxford University Press, Oxford, UK, 1977.

[12] V. Bulmer-Thomas, InputCoutput Analysis in Developing Countries: Sources, Methods and Applications, Wiley, Hoboken, NJ, USA, 1982.

[13] W. Leontief and F. Duchin, The Future Impact of Automation on Workers, Oxford University Press, Oxford, UK, 1985.

[14] W. Peterson, Advances in Input-Output analysis:Technology, Planning, and Development, Oxford University Press, Oxford, UK, 1991.

[15] R. E. Miller and P. D. Blair, Input-output Analysis: Foundations and Extensions, Prentice-Hall, Englewood Cliffs, NJ, USA, 1985.

[16] L.-K. Hua, "On the mathematical theory of globally optimal planned economic systems," Proceedings of the National Academy of Science, USA, vol. 81, no. 20, pp. 6549-6553, 1984.

[17] L.-K. Hua, "Mathematical theory of large-scale optimization in planned economy (I)," Chinese Science Bulletin, vol. 29, no. 12, pp. 6-11, 1984, in Chinese.

[18] L.-K. Hua, "Mathematical theory of large-scale optimization in planned economy (II-III)," Chinese Science Bulletin, vol. 29, no. 13, pp. 769-772, 1984, in Chinese.

[19] L.-K. Hua, "Mathematical theory of large-scale optimization in planned economy (IV-VI)," Chinese Science Bulletin, vol. 29, no. 16, pp. 961-965, 1984, in Chinese.

[20] L.-K. Hua, "Mathematical theory of large-scale optimization in planned economy (VII)," Chinese Science Bulletin, vol. 29, no. 18, pp. 1089-1092, 1984, in Chinese.

[21] L.-K. Hua, "Mathematical theory of large-scale optimization in planned economy (VIII)," Chinese Science Bulletin, vol. 29, no. 21, pp. 1281-1282, 1984, in Chinese.

[22] L.-K. Hua, "Mathematical theory of large-scale optimization in planned economy (IX)," Chinese Science Bulletin, vol. 30, no. 1, pp. 1-2, 1985, in Chinese.

[23] L.-K. Hua, "Mathematical theory of large-scale optimization in planned economy (X)," Chinese Science Bulletin, vol. 30, no. 9, pp. 641-645, 1985, in Chinese.

[24] O. Blanchard, The Need for Different Classes of Macroeconomic Models, Blog Post, Peterson Institute for International Economics, Washington, D.C, USA, 2017, https://piie.com/blogs/.

[25] A. P. Schinnar, "A multidimensional accounting model for demographic and economic planning interactions," Environment and Planning A: Economy and Space, vol. 8, no. 4, pp. 455-475, 1976.

[26] G. Chinchilnisky, G. Heal, and A. Beltratti, "The green golden rule," Economics Letters, vol. 49, pp. 174-179, 1995.

[27] G. Chinchilnisky, "What is sustainable development?" Land Economics, vol. 73, pp. 476-491, 1997.

[28] C. Cinzia, J. Raja, and M. Simone, "Multi-criteria decision analysis with goal programming in engineering, management 
and social sciences: a state-of-the art review," Annals of Operations Research, vol. 251, 2015.

[29] R. M. Solow and P. A. Samuelson, "Balanced growth under constant returns to scale," Econometrica, vol. 21, no. 3, pp. 412-424, 1953.

[30] A. H. Roger and R. Johnson, Matrix Analysis, Cambridge University Press, London, UK, 2nd edition, 2013. 